\title{
Hysteresis phenomena in transverse galloping of triangular cross-section bodies
}

\author{
G. Alonso*, A. Sanz-Lobera, J. Meseguer \\ IDR/UPM, E.T.S.I. Aeronáuticos, Universidad Politécnica de Madrid, E-28040 Madrid, Spain
}

\begin{abstract}
A B S T R A C T
Transverse galloping is a type of aeroelastic instability characterised by large amplitude, low frequency oscillation of a structure in the direction normal to the mean wind direction. It normally appears in bodies with small stiffness and structural damping, provided the incident flow velocity is high enough. In the simplest approach transverse galloping can be considered as a one-degree-of-freedom oscillator subjected to aerodynamic forces, which in turn can be described by using a quasi-steady description. In this frame it has been demonstrated that hysteresis phenomena in transverse galloping is related to the existence of inflection points in the curve giving the dependence with the angle of attack of the aerodynamic coefficient normal to the incident flow. Aiming at experimentally checking such a relationship between these inflection points and hysteresis, wind tunnel experiments have been conducted. Experiments have been restricted to isosceles triangular cross-section bodies, whose galloping behaviour is well documented. Experimental results show that, according to theoretical predictions, hysteresis takes place at the angles of attack where there are inflection points in the lift coefficient curve, provided that the body is prone to gallop at these angles of attack.
\end{abstract}

\section{Introduction}

Galloping occurs due to the aerodynamic forces that are induced by the transverse motions of the structure. The aerodynamic self-excited forces act in the direction of the transverse motion resulting in negative damping, which increases the amplitude of the transverse motion until it settles down to a limited cycle.

Galloping-induced oscillations are caused by forces which act on a structural element as it is subjected to periodic variations in the angle of attack of the wind flow. Usually the periodically varying angle of attack is generated by acrosswind oscillation of the structure. The frequency of the oscillations is approximately the same as the natural frequency of the structure, provided that the density of the structure is much higher than the flow density.

In the last decades galloping has focused the attention of many researches, and large efforts have been devoted to study the galloping features of many bodies having different cross-sections. The influence of relevant parameters like the incident turbulence (Novak and Tanaka, 1974; Li et al., 1998; Ziller and Ruscheweyh, 1997, Hémon et al., 2001; Hémon and Santi, 2002), the geometry of the cross section (Ruecheweyh et al., 1996; Kawai, 1998; Luo et al., 1998; Gjelstrup and Georgakis, 2011), the Reynolds number (Sen and Mittal, 2011; Joly et al., 2012) or the hysteresis phenomenon (Parkinson and Brooks, 1961; Parkinson and Smith,1964; Luo et al., 2003; Ng et al., 2005, Vio et al., 2007; Barrero-Gil et al., 2009;

\footnotetext{
*Corresponding author. Tel.:/fax: +34 913366353.

E-mail address: gustavo.alonso@upm.es (G. Alonso).
} 
Dunnmon et al., 2011) have been treated. Although most of the effort in galloping research has been concentrated in bodies with square or rectangular cross-sections, prismatic bodies with other cross-sectional shapes have been also considered (Blevins, 1990; Naudascher and Rockwell, 1994; Alonso and Meseguer, 2006; Alonso et al., 2005, 2007, 2009, 2010).

The description of the behaviour of a structure under the action of an incident flow is an extremely complex problem, whose theoretical analysis requires the settling of simplified models for making the analysis more affordable. In the derivation of such a simplified mathematical model some simplifying assumptions are needed, the ones giving to the simplest model being that the structure is described as a linear oscillator of one degree of freedom, that the structure is sufficiently slender to consider two-dimensional flow, and finally that the incident flow is free of turbulence. Under these conditions, focussing the attention on triangular cross-section bodies, let us consider a structure that at rest is oriented at given angle of attack $\alpha$ with respect to the incident flow. Assuming the structure oscillating along $y$ axis direction within an uniform flow with velocity $U$ (Fig. 1), the relative velocity between the fluid and the body is $V=\left[U^{2}+(\mathrm{d} y / \mathrm{d} t)^{2}\right]^{1 / 2}$, and the angle of attack due to oscillation is $\Delta \alpha=\operatorname{atan}((\mathrm{d} y / \mathrm{d} t) / U)$. Therefore, drag $d(\alpha+\Delta \alpha)$ and lift $l(\alpha+\Delta \alpha)$ are: $d(\alpha+\Delta \alpha)=$ $(1 / 2) \rho V^{2} b c_{d}(\alpha+\Delta \alpha)$, and $l(\alpha+\Delta \alpha)=(1 / 2) \rho V^{2} b c_{i}(\alpha+\Delta \alpha)$, respectively, where $\rho$ stands for the fluid density, $b$ for a characteristic length of the structure, $c_{d}$ for the drag coefficient and $c_{l}$ for the lift coefficient. The projection of those forces in $y$ axis direction is $f_{y}(\alpha+\Delta \alpha)=(1 / 2) \rho V^{2} b\left[c_{d}(\alpha+\Delta \alpha) \sin \Delta \alpha+c_{l}(\alpha+\Delta \alpha) \cos \Delta \alpha\right]$, thence, since $V^{2}=U^{2}\left[1+(\mathrm{d} y / \mathrm{d} t)^{2} /\right.$ $\left.U^{2}\right]=U^{2}\left[1+(\Delta \alpha)^{2}\right]$ the dimensionless coefficient of the aerodynamic force in the normal direction to the incident flow becomes $c_{f}(\alpha+\Delta \alpha)=\left[1+(\Delta \alpha)^{2}\right] \cdot\left[c_{d}(\alpha+\Delta \alpha) \sin \Delta \alpha+c_{l}(\alpha+\Delta \alpha) \cos \Delta \alpha\right]$.

Therefore, the equation of the movement of the body, assuming that the mass per unit length is $m$, and that it is elastically mounted on a support with lineal damping characterised by a dimensionless structural damping coefficient $\zeta_{y}$ and an undamped natural frequency $\omega_{\eta}$, in dimensionless variables reads:

$$
\frac{\mathrm{d}^{2} \eta}{\mathrm{d} \tau^{2}}+2 \zeta_{\eta} \frac{\mathrm{d} \eta}{\mathrm{d} \tau}+\eta=\mu U_{\mathrm{r}}^{2} c_{f}(\alpha+\Delta \alpha)
$$

where $\eta$ denotes the dimensionless vertical position, $\eta=y / b, \tau$ is the dimensionless time, $\tau=\omega_{\eta} t, \mu$ is the dimensionless mass of the structure per unit length, $\mu=\rho b^{2} /(2 m)$, and $U_{r}$ is the reduced velocity, $U_{r}=U /\left(\omega_{\eta} b\right)$.

From Eq. (1) the main features concerning galloping can be derived. In effect, assuming the angles of attack due to oscillation to be small enough $(\Delta \alpha i \ll 1)$, if the aerodynamic force coefficient is expanded in powers of $\Delta \alpha$ (which is proportional to $\mathrm{d} \eta / \mathrm{d} \tau)$ :

$$
c_{f}=\sum_{n=0}^{\infty} a_{n}(\Delta \alpha)^{n},
$$

the linear term of the expansion can be considered as a contribution to the total damping of the system (aerodynamic damping). Then, since the mechanical damping is generally positive, instability can only occur if the aerodynamic damping is negative, thus the well-known Den Hartog stability criterion (Den Hartog, 1956) for the potential susceptibility of a structure to galloping starting from a given equilibrium position is obtained: $\mathrm{d} c_{l}(\alpha) / \mathrm{d} \alpha+c_{d}(\alpha)<0$.

Once the function $c_{f}(\alpha)$ is known, Eq. (1) can be solved either numerically or by asymptotic methods if the nonlinear aerodynamic term is small. In the case that both aerodynamic and damping forces are small compared with inertia and stiffness forces, solutions of Eq. (1) tend to a limit cycle of quasi-harmonic oscillations (this behaviour of the structure is quite usual because their mean densities are much higher than the density of the surrounding fluid). Depending on the shape of the curve $c_{f}(\alpha)$ some hysteresis phenomena can appear, the hysteresis being characterised by the existence of a solution that can alternately reach different cycles limits (the amplitude of the limit cycle of oscillation can be different, depending on whether the reduced velocity is increasing or decreasing).

In a recently published paper (Barrero-Gil et al., 2009), the dynamic behaviour of galloping bodies has been analytically analysed by using a perturbation (Krylov-Bogoliubov) method, and the solutions corresponding to a variety of $c_{f}(\alpha)$ curves

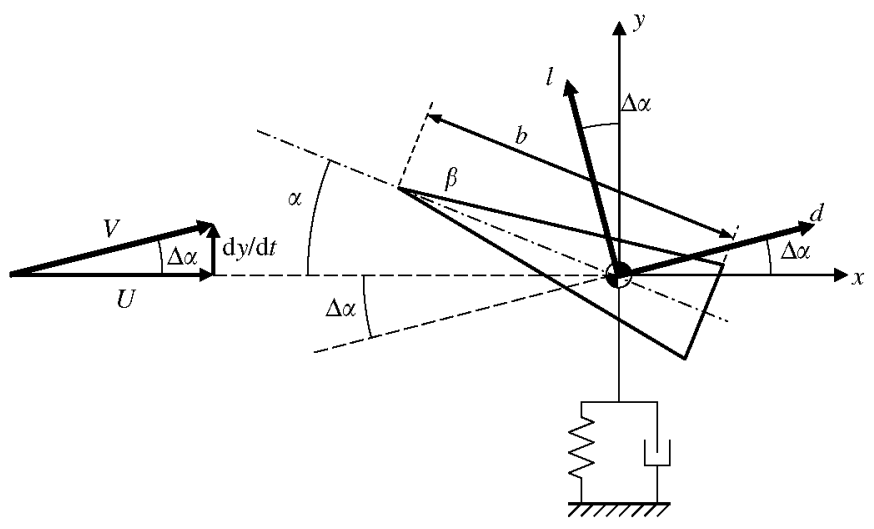

Fig. 1. Definition of the geometry of the isosceles triangular bodies and of the parameters involved in the one degree of freedom galloping model. 
typical of bluff bodies were studied in order to clarify the correlation between the number of inflection points in the $c_{f}(\alpha)$ curves and the hysteresis, the main conclusion being that the number and position of the inflection points determine the regions where hysteresis in the galloping motion can appear. Thus, since $\Delta \alpha$ is small, in a first attempt it is $c_{f}(\alpha) \approx c_{1}(\alpha)$, and then the existence of an inflection point implies $\mathrm{d}^{2} c_{l}(\alpha) / \mathrm{d} \alpha \alpha^{2}=0$.

The hysteresis phenomenon of two dimensional bodies having isosceles triangular cross-sections (Fig. 1) when subjected to cross-flow translational galloping vibration has been analysed through wind tunnel experiments. Triangular cross-section bodies have been chosen because these bodies have been extensively studied in the past (Alonso and Meseguer, 2006; Alonso et al., 2005, 2007; Iungo and Buresti, 2009), so that a large amount of information is now available. In order to clarify the influence of the geometry on the hysteresis, six different triangular cross-sections have been considered, so that the hysteresis map in the angle of attack, triangle main vertex angle $(\alpha, \beta)$, has been experimentally studied.

\section{Theoretical background}

According to the results already published (Alonso and Meseguer, 2006; Alonso et al., 2005, 2007) the stability to transverse translational galloping of triangular cross-section cylinders are both cross-sectional geometry and angle of attack dependent. Available experimental results also show that configurations which are stable at a certain reduced incident wind velocity become unstable when that velocity is increased beyond a critical value that depends on the configuration (cross-section geometry and angle of attack). Isosceles triangular cross-section prisms may present galloping instability (if the appropriate critical incident wind velocity is reached) in configurations where the main vertex of the triangle is almost pointing the incident flow (at angles of attack higher than the ones where the triangular profile stalls, around $\alpha=20^{\circ}$ ) or in configurations with the base of the triangle facing the wind flow $\left(\alpha \approx 180^{\circ}\right.$, the flow approaching clearly a bluff body), covering the angles of attack interval $\alpha \approx 180^{\circ} \pm \beta / 2$, basically when the wind flow is aligned with the lateral sides of the triangle (Alonso et al., 2005). Another less critical instability region appears for intermediate values of $\alpha$, between $50^{\circ}$ and $90^{\circ}$, provided the vertex angle $\beta$ is high enough (Fig. 2).

However, as already stated this is not the only criterion to be considered for hysteresis, in addition the $c_{l}(\alpha)$ curve must present an inflection point within the range of values of the angle of attack where galloping can takes place. Fig. 3 shows the variation with the angle of attack $\alpha$ of the functions $c_{l}(\alpha), d c_{i}(\alpha) / \mathrm{d} \alpha+c_{d}(\alpha)$ and $\mathrm{d}^{2} c_{l}(\alpha) / \mathrm{d} \alpha^{2}$ corresponding to the six triangle geometries under consideration $\left(\beta=10^{\circ}, 20^{\circ}, 30^{\circ}, 40^{\circ}, 50^{\circ}\right.$ and $\left.60^{\circ}\right)$. In these plots inflection points are identified by the condition $\mathrm{d}^{2} c_{l}(\alpha) / \mathrm{d} \alpha^{2}=0$, thus only angles of attack close to these inflection points lying within galloping instability regions have been tested. These points where hysteresis can appear are identified with thick striped lines in Fig. 2.

\section{Experimental set-up}

To perform the experiments reported in this paper an open circuit wind tunnel has been used. Wind tunnel test chamber is $0.15 \mathrm{~m}$ width, $0.90 \mathrm{~m}$ high and $1.20 \mathrm{~m}$ long. Wind velocity profile at the model test section is uniform within $\pm 1 \%$, the turbulence intensity being around $2 \%$. The wind tunnel is powered by an electric motor with variable frequency

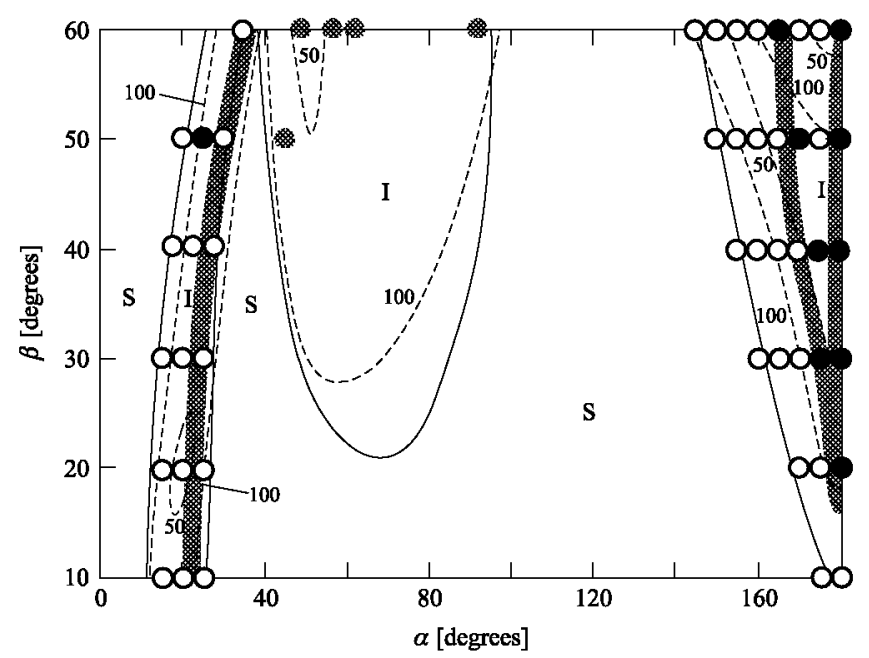

Fig. 2. Stability diagram in the angle of attack-main vertex angle plane $(\alpha, \beta)$ based on dynamic test results. Numbers on the curves (dashed lines) indicate the value of the reduced incident wind velocity $U_{r}$. Note that the stability diagram is symmetric with respect to $\alpha=180^{\circ}$. Solid line represents the boundaries of the unstable region according to static Den Hartog criterion (Alonso et al., 2005). Striped zones indicate the regions where hysteresis can appear. Circles identify the tested configurations: a black circle indicates that hysteresis has been detected, and a white one that this phenomenon has not been observed. 

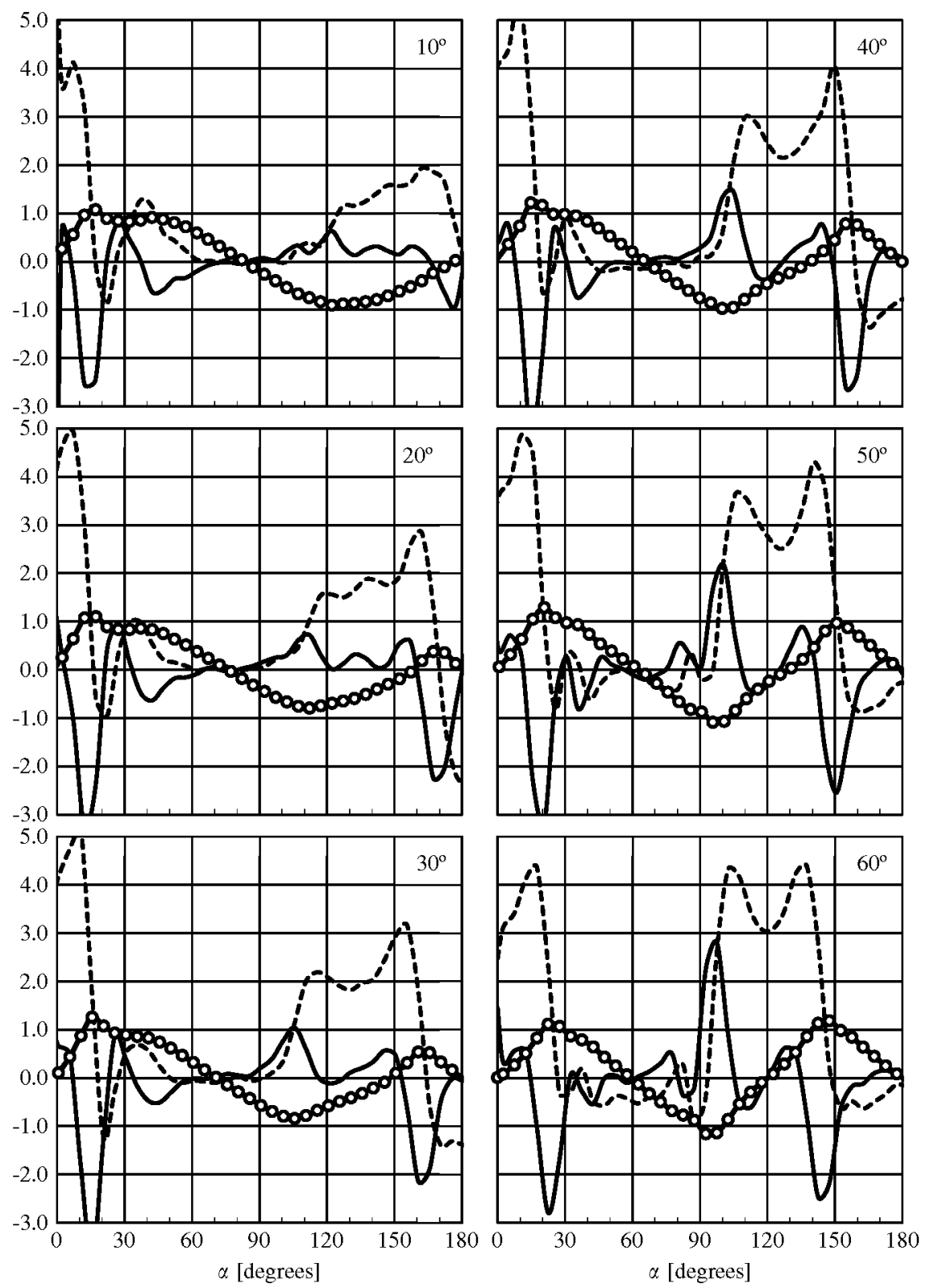

Fig. 3. Variation with the angle of attack, $\alpha$, of the lift coefficient, $c_{l}$ (white circles), the Den Hartog function, d $c_{l} / \mathrm{d} \alpha+c_{d}$ (dashed line), and the function $\left(\mathrm{d}^{2} c_{l} / \mathrm{d} \alpha^{2}\right) / 10$ (solid line). Note that, due to scale constraints, $\left(\mathrm{d}^{2} c_{l} / \mathrm{d} \alpha^{2}\right) / 10$ instead of $\mathrm{d}^{2} c_{l} / \mathrm{d} \alpha^{2}$ has been represented. Experimental results correspond to triangular cross-section bodies with main vertex angle $\beta$ ranging from $10^{\circ}$ to $60^{\circ}$, as indicated in the plots. Note that the curve $c_{l}(\alpha)$ as well as $\mathrm{d}^{2} c_{l} / \mathrm{d} \alpha^{2}$ are anti-symmetric with respect to $\alpha=180^{\circ}$.

drive, which provides a better control on the wind velocity. The wind velocity of the stream at the test section of the wind tunnel was in the range from 7 to $26 \mathrm{~m} / \mathrm{s}$, which provides Reynolds numbers ranging from $10^{4}$ to $2.6 \times 10^{5}$, based on the model characteristic length $b$ (Fig. 1).

Six isosceles triangular cross-section prisms were tested, the main vertex ranging from $\beta=10^{\circ}$ to $\beta=60^{\circ}$ in $10^{\circ}$ steps. All triangles share identical maximum characteristic length $b=0.10 \mathrm{~m}$, the base of the triangle being $2 b \tan (\beta / 2)$. The model span is $0.145 \mathrm{~m}$.

As sketched in Fig. 4, the selected triangular prism (A) is attached to a rod $8 \mathrm{~mm}$ in diameter through an appropriated screw; the rod is fixed to the extreme side of a mechanism (C), in such a way that the prism angle of attack, $\alpha$, can be set with $\pm 0.5^{\circ}$ accuracy (D). The mechanism consists of two long parallel beams which are fixed to a reference frame through ball bearings. At the opposite ends of the long parallel beams is located the vertical small beam that supports the prisms, this small beam being joined to the long ones through ball bearings also. This mechanism allows the vertical (normal to 


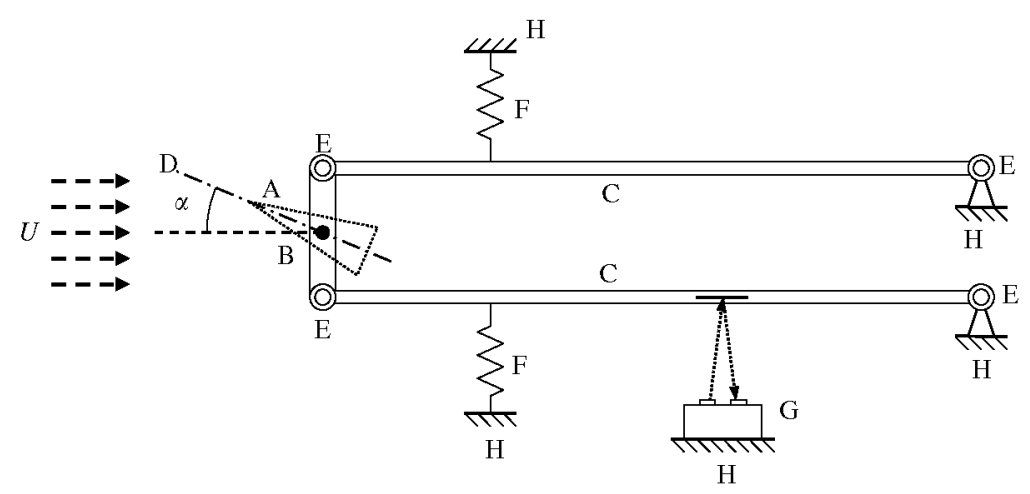

Fig. 4. Sketch of the dynamic test lay-out: prism model (A), supporting rod (B), oscillating parallel beams (C), angle of attack scale (D), rotating ball bearings $(E)$, springs $(F)$, laser vibrometer $(G)$, and reference frame $(H)$. All the elements are placed in a tight chamber outside the wind tunnel test, except the prism, which is inside the test chamber, and the supporting rod that joint the prism to the oscillating mechanism, which is partly inside the test chamber and partly outside, passing through a vertical slot made at the test chamber wall.

Table 1

Mechanical properties of the prisms: mass, structural damping coefficient, natural frequency and Scruton number $S c=2 m \xi / \rho b^{2}$.

\begin{tabular}{lllll}
\hline Triangle main angle, $\beta$ & Mass, $m[\mathrm{~kg} / \mathrm{m}]$ & Structural damping coefficient, $\zeta$ & Natural frequency, $\omega_{n}[\mathrm{~Hz}]$ & Sc \\
\hline $10^{\circ}$ & 2.15 & 0.008 & 2.5 & 3 \\
$20^{\circ}$ & 2.15 & 0.008 & 2.5 & 3 \\
$30^{\circ}$ & 2.35 & 0.008 & 2.3 & 3 \\
$40^{\circ}$ & 2.55 & 0.010 & 2.1 & 4 \\
$50^{\circ}$ & 2.65 & 0.014 & 2.0 & 6 \\
$60^{\circ}$ & 2.65 & 0.014 & 2.0 & 6 \\
\hline
\end{tabular}

wind) motion of the prisms. In addition, there are two springs (F), allowing thus to control the frequency of the oscillation in the normal to flow direction. Note that with this testing device oscillation is purely translational.

The normal to flow displacement of the prisms are recorded using a laser vibrometer M7/L100 from MEL Instrument, with a range of $100 \mathrm{~mm}$, a resolution of $6 \mu \mathrm{m}$ and a sampling frequency of $54 \mathrm{kHz}$. This equipment is anchored to the fixed frame, the laser beam being directed toward a reflecting surface placed on the lower long beam. Measurements were registered at $800 \mathrm{~Hz}$ sampling rate during $25 \mathrm{~s}$ with a $12 \mathrm{bit} \mathrm{A} / \mathrm{D}$ board (from Keithley instruments). Post-processing of the recorded signals gives plots of normal to flow displacement versus time.

Experimental sequence is as follows: once a selected model is fixed to the supporting rod of the holding system, and the chosen reference angle of attack $\alpha$ is set, the experimental sequence runs almost automatically. The wind tunnel is switched on at a small velocity and the output from the laser vibrometer is recorded during $25 \mathrm{~s}$, after a given period of time (typically around $30 \mathrm{~s}$ ), to let the oscillations reach the limit cycle. Then the wind tunnel velocity is increased and, after waiting again $30 \mathrm{~s}$ to overpass the transient period, the new output from the laser vibrometer is recorded. Preliminary tests were performed to determine that a time of $30 \mathrm{~s}$ after changing the incident wind velocity was enough to let the configuration reach the limit cycle, with a maximum deviation of $5 \%$ in the amplitude of the limit cycle during the following $25 \mathrm{~s}$. The procedure described before is repeated until the entire range of wind velocities is covered (from $7 \mathrm{~m} / \mathrm{s}$ to $26 \mathrm{~m} / \mathrm{s}$ at small steps, typically $1 \mathrm{~m} / \mathrm{s}$ ), and then the process is repeated again but now with decreasing wind tunnel velocities (from $26 \mathrm{~m} / \mathrm{s}$ to $7 \mathrm{~m} / \mathrm{s}$, also at small steps). Static and dynamic pressures inside test chamber were measured with an Air Flow 048 Pitot tube connected to a Schaewitz Lucas P-3061-2WD pressure transducer.

The natural frequency $\omega_{\eta}$ and the structural damping coefficient $\zeta_{\eta}$ of each one of the models tested have been measured using the same experimental configuration, by letting the model vibrate freely in no wind conditions. Corresponding values are given in Table 1 , together with the mass and the Scruton number, defined as Sc $=2 m \zeta / \rho b^{2}$. It must be pointed out that the stiffness of the models has been chosen low enough in order to find critical galloping velocities within the range of velocities allowed by the wind tunnel used in the tests, and also to get natural frequencies below the vortex shedding frequencies (decoupling therefore both phenomena, galloping and vortex shedding). For the same reason, the testing device has been designed with a structural damping as low as possible, since it is not the purpose of this paper to evaluate the influence of the mechanical properties in the galloping stability.

Once the whole range of velocities has been tested (both in the increasing velocity sense and in the decreasing one), a new value of the angle of attack of reference is set, and the process is repeated again. The same procedure being applied to each one of the triangular cross-section bodies under consideration. The selection on the reference angles of attack has been done taking into account the existing knowledge on the galloping behaviour of these bodies (Fig. 2). 


\section{Results discussion}

As above stated, hysteresis requires that for a given angle of attack and triangle geometry $(\alpha, \beta)$ the body must be prone to gallop (that means that the Den Hartog stability criterion must be fulfilled) and that close to the angle of attack under consideration the $c_{f}(\alpha) \approx c_{1}(\alpha)$ curve present an inflection point. Therefore, there are two conditions to be fulfilled simultaneously: the Den Hartog criterion, which implies $\mathrm{d} c_{l}(\alpha) / \mathrm{d} \alpha+c_{d}(\alpha)<0$, and the existence of a inflection point, which roughly speaking implies $\mathrm{d}^{2} c_{l}(\alpha) / \mathrm{d} \alpha^{2}=0$.

From the temporal series of normal to wind displacements, $a(t)$, corresponding to each wind velocity $U$ and tests configuration, identified by the angles $\beta$ and $\alpha$, the dimensionless root mean square of the lateral displacement $A=a_{\operatorname{rnns}} / b$ results, where $a_{\mathrm{rms}}$ is the root mean square value of the $a(t)$ series and $b$ the characteristic length defined in Fig. 1. Besides, the reduced velocity $U_{r}=U /\left(\omega_{\eta} b\right)$ is also calculated.

The results obtained, $A$ versus $U_{r}$, for some selected configurations are depicted in Figs. 5 and 6 , and a summary of the hysteresis behaviour of tested triangular bodies is presented in Fig. 2. Concerning Fig. 5, at is can be observed the hysteresis depends on the angle of attack, this phenomenon appearing only around the values of $\alpha$ where an inflection point exists.

31 different configurations, spread on the plane $\alpha-\beta$, in the areas of interest (where hysteresis can appear), have been tested. These areas are angles of attack close to $\alpha=30^{\circ}$ and close to $\alpha=180^{\circ}$. The values of $\alpha$ were selected with a difference of $5^{\circ}$ between two consecutive values, in order to clearly separate the dynamic responses of two adjacent configurations. Note that in the experimental procedure there are many parameters involved, and although all along the experimentation
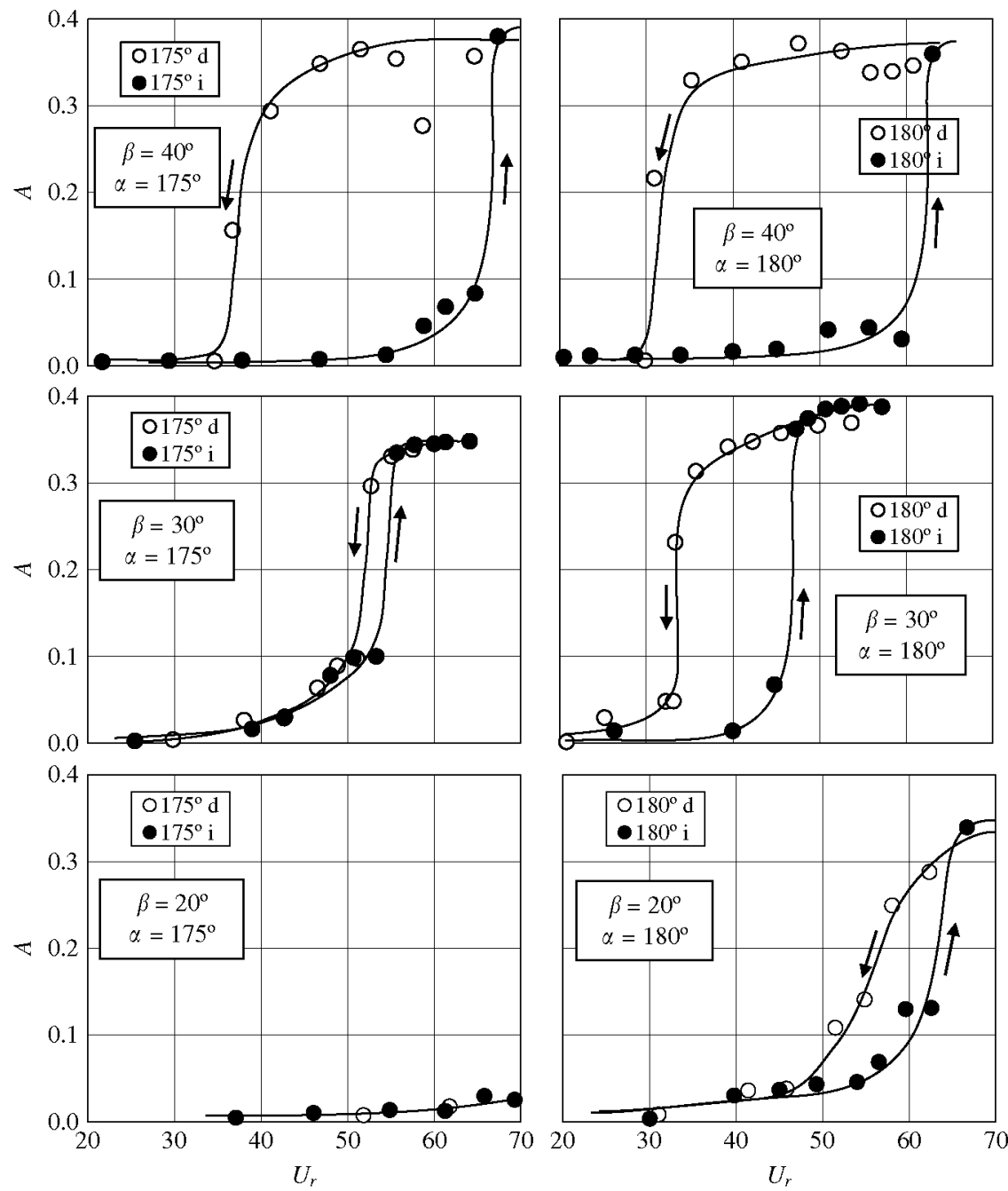

Fig. 5. Variation with the reduced velocity, $U_{r}$ of the dimensionless root mean square oscillation amplitude, $A$. Experimental results correspond to triangular cross-section bodies with main vertex angles $\beta$ and angles of attack $\alpha$, as indicated in the plots. Black circles indicate results obtained by increasing the reduced velocity, whereas white ones indicate results obtained by decreasing the reduced velocity. 

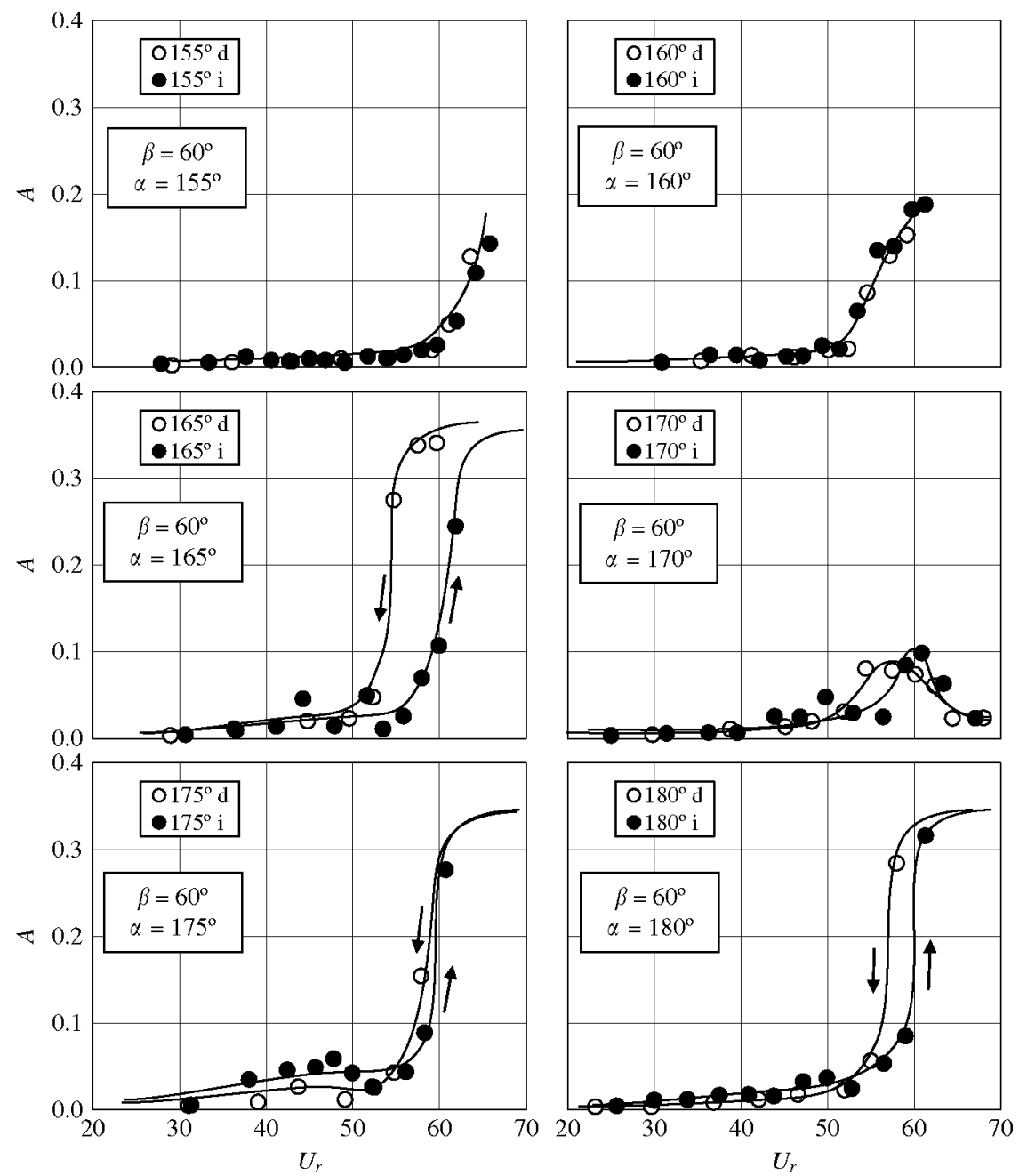

Fig. 6. Variation with the reduced velocity, $U_{r}$, of the dimensionless root mean square oscillation amplitude, $A$. Experimental results correspond to triangular cross-section bodies with main vertex angle $\beta=60^{\circ}$ and angles of attack $\alpha$, as indicated in the plots. Black circles indicate results obtained by increasing the reduced velocity, whereas white ones indicate results obtained by decreasing the reduced velocity.

these parameters have been carefully controlled, the hysteresis itself is a noisy phenomenon influenced by many other variables which are extremely difficult to control, such as small variations in the incident flow conditions (mean velocity and turbulence intensity) or mechanical inaccuracies in the experimental apparatus. It must be stated that the existence of hysteresis on the dynamic response of a given configuration has been considered to exist only when the $A$ versus $U_{r}$ curves obtained during the increasing incident flow velocity phase and the decreasing one are clearly different.

It seems, however, that this $5^{\circ}$ separation into two consecutive values of the angle of attack is a too large step in tests with low values of $\alpha$ (in the region close to $\alpha=30^{\circ}$ ). This would explain why no hysteresis has been detected in this area, except for triangles with $\beta=50^{\circ}$, see Fig. 2. There is another explanation based also on the limitations of the test setup. In effect, as it can be observed in Fig. 2 , in this region $\left(\alpha \approx 30^{\circ}\right)$ galloping instability is associated with large values of the reduced velocity $U_{R}$ (see also Alonso et al., 2005). Therefore, since $U=U_{R} \omega_{n} b$, for a given value of the reduced velocity, the wind speed at the test chamber increases as the natural frequency $\omega_{n}$ does or, according to data shown in Table 1 , as the triangle main vertex angle decreases. Thence, in some configurations the maximum wind tunnel air speed was reached before hysteresis appears.

The situation is much more clear in the region close to $\alpha=180^{\circ}$, because for this value of the angle of attack all the configurations tested are prone to show hysteresis provided that $\beta>10^{\circ}$. However, it must be stressed that $\alpha=180^{\circ}$ configurations become stable when the vertex angle $\beta$ becomes high enough, so that no galloping occurs at $\alpha=180^{\circ}$ for $\beta>70^{\circ}$ (Alonso and Meseguer, 2006). This behaviour can be observed in Fig. 2: triangles with $\beta=10^{\circ}$ do not show hysteresis at $\alpha=180^{\circ}$, the magnitude of the increasing-decreasing velocity loop growing as the vertex angle grows (Fig. 5). The hysteresis becomes maximum for $\beta \approx 40^{\circ}$, and then it decreases as the value of $\beta$ grows until it disappears for $\beta \approx 70^{\circ}$ (see the response of $\beta=60^{\circ}$ triangles close to $\alpha=180^{\circ}$ in Fig. 6). 
Obviously, it can be expected that the hysteresis be less pronounced as the distance to the inflection point increases. This behaviour is shown in the plot included in Fig. 6, where the response of equilateral triangles $\left(\beta=60^{\circ}\right)$ at angles of attack ranging from $\alpha=155^{\circ}$ to $\alpha=180^{\circ}$ are represented (according to Fig. 3 these triangles have $\mathrm{d}^{2} c_{l} / \mathrm{d}^{2}=0$ at $\alpha \approx 165^{\circ}$ and at $\alpha=180^{\circ}$ ).

Finally, the influence of the free stream turbulence is not considered relevant for this type of geometries with sharp edges, because at any angle of attack the incident flow separates at any of the vertex of the triangle, provided that these vertexes are sharp enough, and from then the flow becomes turbulent, even if the incident flow were initially laminar. This effect was proved by the authors (Alonso et al., 2007) for these same triangles.

\section{Conclusions}

Transverse galloping of triangular cross-section two dimensional bodies has been experimentally analysed focusing the attention on the appearance of hysteresis phenomena in the dynamic response. Based on simple criteria the possibility of hysteresis has been defined (the tested configuration must be prone to gallop according to Den Hartog conclusions and an inflection point must exists in the lift coefficient versus angle of attack curve), which has been experimentally checked in the case of triangular prisms. Experimental results show a good agreement with the theoretical predictions of those simple galloping criteria for angles of attack close to $\alpha=180^{\circ}$, when the base of the triangles are facing the incident flow. In the other region of transverse galloping instability where hysteresis should appear, $\alpha$ around $30^{\circ}$, being the size of the region of the same order of magnitude of the interval chosen to change the angle of attack in the test set-up, the tests reproduce the theoretical result only in one of the configurations. Therefore it can be concluded that the absence of hysteresis in the $\alpha \approx 30^{\circ}$ region is due to the mechanical limitations of the experimental facility rather than to a real absence of hysteresis (which probably will appear at higher wind velocities than those tested).

\section{Acknowledgement}

The authors wish to thank Mr. Barrero-Gil for his helpful comments.

\section{References}

Alonso, G., Meseguer, J., 2006. A parametric study of the galloping instability of triangular cross-section bodies. Journal of Wind Engineering and Industrial Aerodynamics $94,241-253$

Alonso, G., Meseguer, J., Pérez-Grande, I., 2005. Galloping oscillations of two-dimensional triangular cross-sectional bodies. Experiments in Fluids 38 $789-795$.

Alonso, G., Meseguer, J., Pérez-Grande, I., 2007. Galloping stability of triangular cross-sectional bodies: a systematic approach. Journal of Wind Engineering and Industrial Aerodynamics $95,928-940$.

Alonso, G., Meseguer, J., Sanz-Andrés, A., Valero, E., 2010. On the galloping instability of two-dimensional bodies having elliptical cross sections. Journal of Wind Engineering and Industrial Aerodynamics $98,438-448$.

Alonso, G., Valero, E., Meseguer, J., 2009. An analysis on the dependence on cross section geometry of galloping stability of two-dimensional bodies having either biconvex or rhomboidal cross sections. European Journal of Mechanics-B Fluids 28, 328-334.

Barrero-Gil, A., Sanz-Andrés, A., Alonso, G., 2009. Hysteresis in transverse galloping: the role of the inflection points. Journal of Fluids and Structures 25 $1007-1020$.

Blevins, R.D., 1990. In: Flow-Induced Vibrations, 2nd ed. Krieger Publishing Co., Malabar, Florida.

Den Hartog, J.P., 1956. In: Mechanical Vibrations, 4th ed. McGraw-Hill, New York.

Dunnmon, J.A., Stanton, S.C., Mann, B.P., Dowell, E.H., 2011. Power extraction from aeroelastic limit cycle oscillations. Journal of Fluids and Structures 27, 1182-1198.

Gjelstrup, H., Georgakis, C.T., 2011. A quasi-steady 3 degree-of-freedom model for the determination of the onset of bluff body galloping instability. Journal of Fluids and Structures 27, 1021-1034.

Hémon, P., Santi, F., 2002. On the aeroelastic behaviour of rectangular cylinders in cross-flow. Journal of Fluids and Structures 16, 855-889.

Hêmon, P., Santi, F., Schnoerringer, B., Wojciechowski, J., 2001. Influence of free stream turbulence on the movement-induced vibrations of an elongated rectangular cylinder in cross flow. Journal of Wind Engineering and Industrial Aerodynamics 89, 1383-1395.

Iungo, G.V., Buresti, G., 2009. Experimental investigation on the aerodynamic loads and wake flow features of low aspect-ratio triangular prisms at different wind directions. Journal of Fluids and Structures 25, 1119-1135.

Joly, A., Etienne, S., Pelletier, D., 2012. Galloping of square cylinders in cross-flow at low Reynolds numbers. Journal of Fluid and Structures 28 232-243.

Kawai, H., 1998. Effect of comer modifications on aeroelastic instabilities of tall buildings. Journal of Wind Engineering and Industrial Aerodynamics $74-76,719-729$.

Li, Q.S., Fang, J.Q., Geary, A.P., 1998. Evaluation of 2D coupled galloping oscillations of slender structures. Computers \& Structures 66, 513-523.

Luo, S.C., Chew, Y.T., Lee, T.S., Yazdani, M.G., 1998. Stability to translational galloping vibration of cylinders at different mean angles of attack. Journal of Sound and Vibration 215, 1183-1194.

Luo, S.C., Chew, Y.T., Ng, Y.T., 2003. Hysteresis phenomenon in the galloping oscilation of a square cylinder. Journal of Fluids and Structures 18 , $103-118$.

Naudascher, E., Rockwell, D., 1994. Flow-Induced Vibrations: An Engineering Guide. A.A. Balkema, Rotterdam.

Ng, Y.T., Luo, S.C., Chew, Y.T., 2005. On using high-order polynomial curve fits in the quasi-steady theory for square-cylinder galloping. Journal of Fluids and Structures 20,141-146.

Novak, M., Tanaka, H., 1974. Effect of turbulence on galloping instability. ASCE Journal Engineering Mechanics Division 100, 27-47.

Parkinson, P., Brooks, N.P.H., 1961. On the aeroelastic instability of bluff cylinders. Journal of Applied Mechanics 28, 252-258.

Parkinson, P., Smith, J.D., 1964. The square cylinder as an aeroelastic non-linear oscillator. The Quarterly Journal of Mechanics and Applied Mathematics $17,225-239$. 
Ruecheweyh, H., Hortmanns, M., Schnakenberg, C., 1996. Vortex-excited vibrations and galloping of slender elements. Journal of Wind Engineering and Industrial Aerodynamics $65,347-352$.

Sen, S., Mittal, S., 2011. Free vibration of a square cylinder at low Reynolds numbers. Journal of Fluid and Structures $27,875-884$

Vio, G.A., Dimitriadis, G., Cooper, J.E., 2007. Bifurcation analysis and limit cycle oscillation amplitude prediction methods applied to the aeroelastic galloping problem. Journal of Fluids and Structures 23, 983-1011.

Ziller, C., Ruscheweyh, H., 1997. A new approach for determining the onset velocity of galloping instability taking into account the nonlinearity of the aerodynamic damping characteristic. Journal of Wind Engineering and Industrial Aerodynamics 69-71, 303-314. 